\title{
Risk factors of migraine-related brain white matter hyperintensities: an investigation of 186 patients
}

\author{
Anita Trauninger · Eszter Leél-Őssy • David Olayinka Kamson • László Pótó • \\ Mihály Aradi · Ferenc Kövér • Marianna Imre · Hedvig Komáromy • \\ Szilvia Erdélyi-Botor · Ágnes Patzkó • Zoltán Pfund
}

Received: 25 August 2010/Accepted: 23 November 2010/Published online: 18 February 2011

(C) The Author(s) 2011. This article is published with open access at Springerlink.com

\begin{abstract}
Brain white matter hyperintensities are more prevalent in migraine patients than in the general population, but the pathogenesis and the risk factors of these hyperintensities are not fully elucidated. The authors analyzed the routine clinical data of 186 migraine patients who were referred to the Outpatient Headache Department of the Department of Neurology, Medical School, University of Pécs, Hungary between 2007 and 2009: 58 patients with white matter hyperintensities and 128 patients without white matter hyperintensities on 3 T MRI. Significant associations between the presence of white matter hyperintensities and longer disease duration (14.4 vs. 19.9 years, $p=0.004$ ), higher headache frequency (4.1 vs. 5.5 attacks/month, $p=0.017$ ), hyperhomocysteinemia (incidence of hyperintensity is $9 / 9=100 \%, p=0.009$ ) and thyroid gland dysfunction (incidence of hyperintensity is $8 / 14=57.1 \%$, $p=0.038$ ) were found. These data support the theory that both the disease duration and the attack frequency have a key role in the formation of migraine-related brain white matter hyperintensities, but the effects of comorbid diseases may also contribute to the development of the hyperintensities.
\end{abstract}

Keywords Migraine - White matter hyperintensity . Disease duration and attack frequency - Stroke risk factors

A. Trauninger · E. Leél-Őssy · D. O. Kamson ·

S. Erdélyi-Botor · Á. Patzkó · Z. Pfund ( $\square)$

Department of Neurology, University of Pécs,

7623 Pécs, Rét u. 2, Pecs, Hungary

e-mail: zoltan.pfund@aok.pte.hu

\section{Pótó}

Institute of Bioanalysis, University of Pécs, Pecs, Hungary

M. Aradi · F. Kövér · M. Imre · H. Komáromy

Diagnostic Center, Pecs, Hungary

\section{Introduction}

Migraine is a complex disorder of the brain, and its pathomechanism is intensively investigated. Migraine is not as benign a disease as it was thought before the imaging era since migraine is an independent risk factor for deep white matter lesions, silent posterior circulation territory infarcts, and infratentorial hyperintense lesions [1-4]. Migraine patients have an almost fourfold greater risk to develop white matter lesions than non-migraine controls [5], and the prevalence of these lesions is higher in migraine with aura than in migraine without aura [4]. The risk of developing white matter lesions is higher in female migraineurs and in patients with higher attack frequency and longer disease duration $[4,6]$. Furthermore, it seems that brain white matter lesions do not correlate with age [7, 8]. It is also known that beyond the existing relation between migraine and stroke, there is also an association between migraine and coronary heart disease $[9,10]$. Since the risk of these severe complications is low in the general population, it is not possible to identify which migraine patients will develop a cerebrovascular or cardiovascular event [10].

The clinical significance of white matter hyperintensities (WMH) is uncertain and the pathogenesis of these lesions is likely multifactorial. A number of pathophysiological mechanisms have been proposed including attack-related oligemia and focal hypoperfusion [3,11], glutamatergic excitotoxicity $[11,12]$, immune-based white matter demyelination [13], and mitochondrial dysfunction [14]. Several studies considered the role of inflammation as a vascular risk for brain white matter lesions in migraine [15-17]. Elevated C-reactive protein found in migraine patients is a sensitive indicator of active systemic inflammation and a marker of oxidative stress [15]. Increased proinflammatory cytokines (IL-1, IL-6, TNF- $\alpha$ ) have been 
reported during acute migraine attacks, as well as in the interictal periods [16, 17]. Repeated sterile vascular inflammation results in endothelial injury of the cranial blood vessels, cranial arteriopathy, and consequent thrombosis $[18,19]$. It has been suggested that cortical spreading depression causes disruption of the blood brain barrier through a matrix metalloproteinase-9-dependent cascade mechanism which may result in local tissue damage [20]. It is also known that endothelial dysfunction associates with an increased rate of cerebrovascular ischemic events, and genetic factors (such as angiotensinconverting enzyme insertion/deletion, methylenetetrahydrofolate reductase C677T polymorphisms, and von Willebrand factor activity) which increase the susceptibility of endothelial dysfunction have been linked to migraine $[21,22]$.

Although migraineurs, particularly with aura, have a higher cardiovascular risk profile than individuals without migraine [23], the presence of white matter lesions proved to be independent of a history of hypertension, diabetes, smoking, hypercholesterolemia, hyperhomocysteinemia, patent foramen ovale with right-to-left shunt, and oral contraceptive use [5, 24, 25]. Associations with antiphospholipid antibodies (anticardiolipin antibodies, lupus anticoagulans) and abnormal coagulation parameters (antithrombin-III, protein S, protein C) were also not found [26].

Because of the high prevalence of white matter hyperintensities, and the partly contradictory and uncertain etiological data, we have investigated the possible risk factors for subclinical brain white matter hyperintensities in our own migraine patients by analyzing their routine clinical data.

\section{Patients and methods}

Subjects and studies

Subjects included in this study were referred to the Outpatient Headache Department of the Department of Neurology, Medical School, University of Pécs, Hungary between 2007 and 2009. All the patients who were chosen for the study met the criteria of migraine patients as defined by the International Headache Society [27]. Further, patients were also selected based on the lack of major comorbidities, such as hypertension, cardiac disease, diabetes, thyroid gland dysfunction, oncological and haematological diseases, infectious diseases (e.g. HIV, hepatitis), central nervous system demyelination (e.g. multiple sclerosis), and genetically inherited disorders (e.g. CADASIL). Altogether 186 migraine patients were investigated in the study (141 patients without aura, 45 patients with aura, age range $18-58$ years, mean age 36.4 years, SD: 8.9; 156 females and 30 males). Based on the findings of brain magnetic resonance imaging (MRI) studies, patients were divided into two groups: patients with WMH (WMH+ group, $n=58$, mean age 40.2 year, 54 patients had supratentorial hyperintensities, 4 patients had cerebellar hyperintensities) and patients without WMH (WMHgroup, $n=128$, mean age 34.9 year).

All participants underwent a structured clinical examination (history taking, physical examination, blood pressure measurement, serum and urine tests, brain MRI study) to identify comorbid medical disorders. Gender, migraine type, disease duration, attack frequency, history of smoking and taking oral contraceptives, serum cholesterol (reference range $4.00-5.60 \mathrm{mmol} / \mathrm{l}$ ) and low-density lipoprotein (LDL) (reference range $0.00-3.40 \mathrm{mmol} / \mathrm{l}$ ), uric acid (reference range $180-350 \mu \mathrm{mol} / \mathrm{l}$ ), homocysteine levels (reference range $5.00-15.00 \mu \mathrm{mol} / \mathrm{l})$, and thyroid-stimulating hormone levels (reference range $0.27-4.20 \mathrm{mU} / \mathrm{l}$ ) with thyroxine and triiodothyronine when necessary were routinely examined. Subclinical hypo- and hyperthyroidism was diagnosed when peripheral thyroid hormone levels were within normal reference laboratory range but serum thyroid-stimulating hormone levels were mildly elevated or decreased. In all cases when aura symptoms and/or WMH were present, patients were tested for vasculitis (antinuclear antibody, antineutrophil cytoplasmic autoantibody, antiphospholipid antibody, lupus anticoagulant), for Lyme disease (serum ELISA screening with Western blot testing), and for patent foramen ovale (transthoracic and transesophageal echocardiography).

\section{MRI protocol}

The brain MRI examinations were carried out with an MR scanner operating at $3 \mathrm{~T}$ (Siemens Trio Tim, 12 channel head coil). A qualified neuroradiologist who was blinded to migraine diagnosis and clinical data rated the WMH. WMH were considered if visible as hyperintense on T2weighted and FLAIR images, without hypointensity on T1weighted scans, and were larger than $3 \mathrm{~mm}$ [28].

Statistical analysis

Statistical analysis was performed using the SPSS 15.0 statistical package (SPSS Inc., Chicago, IL, USA). Chisquare tests were performed to assess the difference between the $\mathrm{WMH}+$ and $\mathrm{WMH}-$ groups in relation to migraine type, disease duration, attack frequency, gender, smoking, cholesterol, LDL, uric acid, and thyroid-stimulating hormone levels. To examine the disease duration, patient groups were created in which the disease duration increased by 5 years in each group $(0-5,5-10$, etc.). Furthermore, patients were divided into three groups by monthly attack frequency (rare: $0-1$ attacks/month, 
average: $2-7$ attacks/month, very frequent: 8 or more attacks/month). Since homocysteine level was measured only in 60 patients, and thyroid gland dysfunction was found only in 14 patients, a Fisher's exact test was used to assess the difference between the groups. Both the disease duration and attack frequency were used as dichotomous variables when contingency tables were created and evaluated. Chi-square tests and Fisher's exact test were used for these evaluations. The non-parametric Mann-Whitney test was also applied to investigate the role of the disease duration and the attack frequency in the formation of WMH in different groups, including all patients and migraine subgroups (patients without and with aura). In this analysis, the disease duration and the attack frequency were analyzed as continuous variables. Finally, a binary logistic regression was also performed to predict the presence of WMH from a set of independent variables. For this analysis homocysteine was not used due the number of the tested patients. Group comparisons were not performed for oral contraceptives because most of the migraineurs were female and there was contraceptive use in the past and/or current medical history in almost all cases. A value of $p<0.05$ was considered to be significant.

\section{Results}

1. There was no statistical difference between females ( $n=48$ out of $156,30.7 \%$ ) and males ( $n=10$ out of $30,33.3 \%)$, as well as between migraine patients without aura ( $n=42$ out of $141,30.5 \%$ ) and patients with aura ( $n=16$ out of $45,35.6 \%$ ) in relation to the presence of WMH.

2. The number of patients with $\mathrm{WMH}$ increased with the increase of disease duration; in patients with above 20 migraine years hyperintensities were significantly more frequent than in those with less than 20 years of duration (under 20 years $n=28 / 120,23.3 \%$, above 20 years in $n=30 / 66,45.6 \%, p=0.007)$. When examining the association between the disease duration and the presence of WMH according to migraine type, it was found that both migraine patients without and with aura have a longer disease duration with WMH, than patients without WMH (Table 1).

3. Although the proportion of migraine patients with WMH increased with the monthly migraine attack frequency (0-1 attack/month: $n=7 / 37,18.9 \%, 2-7$ attacks/month: $n=36 / 114,31.5 \%, \geq 8$ attacks/month: $n=15 / 35,42.9 \%$ ), there was only a trend towards statistical significance $(p=0.08)$ when the patients were examined in three groups according to their attack frequency. Conversely, when the attack frequency of the $\mathrm{WMH}+$ group was compared to the attack frequency of the WMH - group, a significantly higher attack number was found in the WMH+ group (Table 1). When examining the effect of attack frequency on the presence of brain WMH in migraine subgroups, it was found that migraine patients without aura and with $\mathrm{WMH}$ have a higher attack frequency than patients without aura and without WMH (Table 1). In patients with aura the same tendency was seen, but the difference was not statistically significant (Table 1).

4. WMH did not occur more frequently in smokers ( $n=18 / 52,34.6 \%)$ than in non-smokers $(n=40 / 134$, $29.8 \%$ ), but smoking was significantly associated ( $p=0.001)$ with increased monthly attack frequency ( $<5$ attacks/month: $n=87 / 134,64.9 \%$ in non-smokers, $n=47 / 134,35.0 \%$ in smokers, $\geq 5$ attacks/month: $n=20 / 52,38.4 \%$ in non-smokers, $n=32 / 52,61.5 \%$ in smokers).

Table 1 Differences between the WMH- and WMH+ groups in relation to disease duration and attack frequency in migraine patients without and with aura

\begin{tabular}{|c|c|c|c|c|c|}
\hline & Patient number & Disease duration (years) & $p$ value & Attack frequency (attack/month) & $p$ value \\
\hline \multicolumn{6}{|c|}{ All patients } \\
\hline WMH- & 128 & $14.3 \pm 8.5$ & \multirow[t]{2}{*}{0.004} & $4.1 \pm 3.0$ & \multirow[t]{2}{*}{0.017} \\
\hline WMH+ & 58 & $19.9 \pm 11.6$ & & $5.5 \pm 3.6$ & \\
\hline \multicolumn{6}{|c|}{ Patients without aura } \\
\hline WMH- & 99 & $14.7 \pm 8.7$ & \multirow[t]{2}{*}{0.030} & $4.6 \pm 3.1$ & \multirow[t]{2}{*}{0.016} \\
\hline WMH+ & 42 & $19.7 \pm 11.7$ & & $6.2 \pm 3.6$ & \\
\hline \multicolumn{6}{|c|}{ Patients with aura } \\
\hline WMH- & 29 & $13.1 \pm 7.9$ & \multirow[t]{2}{*}{0.047} & $2.4 \pm 2.4$ & \multirow[t]{2}{*}{0.067} \\
\hline WMH+ & 16 & $20.3 \pm 11.8$ & & $3.5 \pm 3.0$ & \\
\hline
\end{tabular}

$W M H$ - patients without white matter hyperintensities, $W M H+$ patients with white matter hyperintensities

The diseases duration and attack frequency values are presented in mean $\pm 1 \mathrm{SD}$

$p$ values are based on Mann-Whitney statistical analysis 
5. There was a significant relation between abnormally high serum homocysteine levels and the incidence of brain WMH; out of 60 patients tested for serum homocysteine 9 had WMH and homocysteine levels were elevated in all 9 patients $(p=0.009)$.

6. Subclinical hypo- $(n=8)$ and hyperthyroidism $(n=6)$ was detected in 14 patients (14/ $186=7.5 \%$ ). Out of these patients, eight had WMH ( 4 patients in both groups, $8 / 14=57.1 \%$ ). Statistical analysis showed the subclinical thyroid dysfunction occurred significantly more frequently in the $\mathrm{WMH}+$ group than in the WMH- group $(p=0.038)$.

7. Although the statistical analysis did not show an increased risk of WMH in migraineurs with high cholesterol + LDL cholesterol $(p=0.06)$ and high uric acid levels $(p=0.07)$, the cholesterol + LDL cholesterol and uric acid values were found more frequently in the elevated range in the $\mathrm{WMH}+$ group ( $n=18 / 41,43.9 \%$ for cholesterol + LDL cholesterol, $n=7 / 15,46.6 \%$ for uric acid) than in the WMHgroup $(n=40 / 145,27.5 \%$ for cholesterol + LDL cholesterol, $n=51 / 171,29.8 \%$ for uric acid).

8. When the effects of all the predictor variables were examined, only two good predictor variables were found: the disease duration $(p<0.01)$ and the attack frequency $(p<0.05)$ (Table 2). There was no significant effect with all the other variables $(p>0.1)$ (Table 2).

9. An elevated antiphospholipid antibody titer was only found in one patient. Echocardiography showed a tiny patent foramen ovale with right to left shunt in two migraine patients with aura and with brain WMH. There was no patient with pathologic Lyme serology.

\section{Discussion}

In this study, we have investigated possible risk factors for brain WMH based on migraine history and blood tests. To get more accurate data on those factors which may influence the formation of $\mathrm{WMH}$, diseases which can associate with the presence of brain WMH without migraine, including hypertension, were excluded from our study. We found significantly higher hyperintensity incidence in patients with longer migraine duration, higher headache frequency, subclinical hyper- and hypothyroidism, and elevated plasma concentrations of homocysteine, while in the cases of cholesterol and uric acid, there was a trend towards statistical significance. Statistical difference was not found between migraine patients without and with aura in relation to the presence of $\mathrm{WMH}$, but most of the patients in the $\mathrm{WMH}+$ group had only supratentorial signal abnormalities [1]. There are differences between our results
Table 2 Results of binary logistic regression for white matter hyperintensities

\begin{tabular}{lllll}
\hline Examined variables & $p$ & OR & \multicolumn{2}{l}{$95.0 \%$ CI for OR } \\
\cline { 5 - 6 } & & & Lower & Upper \\
\hline Step 1 & & & & \\
Disease duration & 0.090 & 1.046 & 0.993 & 1.103 \\
Attack frequency & 0.018 & 1.164 & 1.026 & 1.321 \\
Age & 0.913 & 0.997 & 0.947 & 1.049 \\
Gender & 0.865 & 1.087 & 0.414 & 2.855 \\
Smoking & 0.249 & 0.584 & 0.234 & 1.457 \\
Thyroid function & 0.289 & 0.637 & 0.277 & 1.465 \\
Cholesterol & 0.135 & 0.498 & 0.200 & 1.242 \\
Uric acid & 0.447 & 0.515 & 0.093 & 2.849 \\
Step 7 & & & & \\
Disease duration & 0.005 & 1.051 & 1.015 & 1.089 \\
Attack frequency & 0.017 & 1.153 & 1.026 & 1.295 \\
\hline
\end{tabular}

The table presents the $p$ value, the odds ratio (OR) and the $95 \%$ confidence interval (CI) for the OR for all of the variables at Step 1 and for the variables that were significant at Step 7 of the backward stepwise elimination procedure

and the previously documented ones, and we suppose that these are based on the limitations of the performed studies due to differences in patient selection and sample size.

The relation between disease duration and attack frequency to the WMH is not surprising if we take into account the pathophysiology of migraine [29]. During the attack several intracranial pathologic processes are detectable, including intracerebral haemodynamic changes, local inflammatory responses, excessive neuronal activation and excitotoxicity, which may all lead to tissue damage [30]. Although there are regional differences and predilection sites for tissue damage, basically, the migraine attack affects the whole brain $[6,30,31]$. It is known that there are differences among migraine patients in relation to the risk of the WMH. The risk depends not only on the disease duration and frequency, but the migraine type, attack duration, and comorbid conditions can also influence it $[4,6]$. The comorbid conditions are mainly routine stroke risk factors and these may lead to tissue damage by direct (e.g. blood vessel endothelium dysfunction, hypercoagulation, embolization) or indirect (e.g. smoking) effects [21, 23, 32-41]. In our study, the occurrence of WMH was not higher in smokers than in non-smokers, but smoking increased the headache frequency, therefore smoking may indirectly cause white matter hyperintensities. These data are consistent with the previously reported ones showing higher prevalence rates for headache amongst smokers compared to non-smokers [32, 33].

Plasma homocysteine concentration is controlled by genetic (MTHFR C677T mutation), nutritional (vitamins 
folate, B6, B12) and acquired (smoking, alcohol consumption, renal diseases, malignancies, inflammation, daily physical activity) factors [34]. It is also known that endothelial asymmetric dimethylarginine has been linked with elevated levels of homocysteine [35]. Conversely, asymmetric dimethylarginine concentrations are substantially elevated by native or oxidized LDL cholesterol [36]. Furthermore, HDL-cholesterol levels were inversely correlated to the homocysteine levels [37]. In this study, we did not examine which factors could lead to an elevation of homocysteine levels, only if the elevation itself was present. Regardless of the provoking factors, elevated total homocysteine concentration is an independent risk factor for recurrent stroke [38] which may result from an endothelial injury and altered coagulant properties of the blood $[39,40]$; however, it is still controversial whether mild hyperhomocysteinemia is a causal factor. It has been found that migraine patients with aura who are homozygotes for methylene-tetrahydrofolate reductase (MTHFR) C677T variant, are at risk for elevated levels of homocysteine, and homocysteine-related endothelial dysfunction may be involved in the initiation and maintenance of migraine [21].

Although subclinical thyroid gland dysfunction was found in few patients in our study, more than half of these patients had brain WMH. Subclinical thyroid gland dysfunction can be a risk factor for WMH in migraine, but currently there is no definite evidence to confirm this. Previously, in a large cross-sectional population-based study, TSH levels were lower among headache patients, especially migraineurs, than in those without headache complaints [41]. It is also known that the endocrine function, including the plasma concentrations of TSH, is altered in chronic migraine patients with high headache frequency and frequent analgesic use [42]. In addition, a significant correlation was found between the duration of the disease and the altered hormonal response [42]. Hyperthyroidism can associate with atrial fibrillation and cardioembolic stroke and may lead to a hypercoagulability state [43]. Hypothyroidism is associated with a worse cardiovascular risk factor profile including elevated cholesterol and lowdensity lipoprotein levels, diastolic hypertension, increased homocysteine and C-reactive protein concentrations, impaired thyroid hormone action on target tissue by smoking, tendency toward decreased fibrinolytic activity in mild and moderate hypothyroidism, and endothelial dysfunction with progression to atherosclerosis [43].

We found WMH more frequently in our migraine patients with elevated serum cholesterol and uric acid levels, and we speculate that if there is a relation between migraine and cholesterol and uric acids levels, this can be based on altered endothelial dysfunction and migraine attack frequency. Hyperlipoproteinemias can be familial in origin but there are drugs and diseases (e.g. oral contraceptives, hypothyroidism) which can cause secondary hyperlipoproteinemias or worsen underlying hyperlipoproteinemic states [44]. High levels of blood lipids and free fatty acids are among the factors involved in triggering migraine headache [45]. The presence of hypercholesterolemia and dyslipidemia in patients with migraine may increase the risk of vascular wall injury. Hypercholesterolemia is associated with an increase in endothelial permeability, the retention of lipoproteins within the intima of blood vessels, inflammatory cell recruitment and foam cell formation filled with oxidative-LDLs, and finally these processes progress to atherosclerotic plaque maturation [46]. Cholesterol crystals, a component of human atherosclerotic plaques, could also cause an inflammatory response and neuronal injury in the brain with persistent activation of microglia and astrocytes via microembolization [47]. Furthermore, a number of epidemiologic data show that an elevated serum uric acid level is a powerful predictor of an increased risk of a cardiovascular event including stroke and silent brain infarcts [48] however, the available data are contradictory. It is entirely plausible that chronic elevations in serum uric acid levels have harmful effects on platelet, smooth muscle, and endothelial function [49], but the neuroprotective, antioxidant effect of high uric acid levels may associate with improved outcome in the peri-ictal period [50].

In conclusion, this study provides additional data on the etiology of migraine-related WMH supporting the former assumptions that a wide range of factors contribute to lesion formation. These include attack-related intracerebral changes and direct or indirect effects of comorbid diseases. Development in understanding the pathophysiology of migraine and the pathology of $\mathrm{WMH}$, the use of effective therapy for migraine attack and prophylaxis, detection and treatment of vascular risk factors, and avoiding smoking may help to prevent the development of the hyperintensities.

Acknowledgments This work was supported by grants from EEA/ Norwegian Financial Mechanism HU 0114- "Save what can be saved"-applied neurological research using high-field magnetic resonance imaging.

\section{Conflict of interest None.}

Open Access This article is distributed under the terms of the Creative Commons Attribution License which permits any use, distribution and reproduction in any medium, provided the original author(s) and source are credited.

\section{References}

1. Kruit MC, van Buchem MA, Hofman PA, Bakkers JT, Terwindt GM, Ferrari MD, Launer LJ (2004) Migraine is a risk factor for subclinical brain lesions. JAMA 291:427-434 
2. Kruit MC, Launer LJ, Ferrari MD, van Buchem MA (2005) Infarcts in the posterior circulation territory in migraine. The population-based MRI CAMERA study. Brain 128:20682077

3. Kruit MC, Launer LJ, Ferrari MD, van Buchem MA (2006) Brain stem and cerebellar hyperintense lesions in migraine. Stroke 37:1109-1112

4. Kruit MC, van Buchem MA, Launer LJ, Terwindt GM, Ferrari MD (2010) Migraine is associated with an increased risk of deep white matter lesions, subclinical posterior circulation infarcts and brain iron accumulations: the population-based MRI CAMERA study. Cephalalgia 30:129-136

5. Swartz RH, Kern RZ (2004) Migraine is associated with magnetic resonance imaging white matter abnormalities: a metaanalysis. Arch Neurol 61:1366-1368

6. Schmitz N, Admiraal-Behloul F, Arkink EB, Kruit MC, Schoonman GG, Ferrari MD, van Buchem MA (2008) Attack frequency and disease duration as indicators for brain damage in migraine. Headache 48:1044-1055

7. Fazekas F, Koch M, Schmidt R, Offenbacher H, Payer F, Freidl W, Lechner H (1992) The prevalence of cerebral damage varies with migraine type: a MRI study. Headache 32:287-291

8. Benedittis GD, Lorenzetti A, Sina C, Bernasconi V (1995) Magnetic resonance imaging in migraine and tension-type headache. Headache 35:264-268

9. Sacco S, Olivieri L, Bastianello S, Carolei A (2006) Comorbid neuropathologies in migraine. J Headache Pain 7:222-230

10. Sacco S, Cerone D, Carolei A (2008) Comorbid neuropathologies in migraine: an update on cerebrovascular and cardiovascular aspects. J Headache Pain 9:237-248

11. Dodick DW, Roarke MC (2007) Crossed cerebellar diaschisis during migraine with prolonged aura: a possible mechanism for cerebellar infarction. Cephalalgia 28:83-86

12. Longoni M, Ferrarese $C$ (2006) Inflammation and excitotoxicity: role in migraine pathogenesis. Neurol Sci Suppl 2:S107-S110

13. Robbins L, Friedman H (1992) MRI in migraineurs. Headache 32:507-508

14. Sparaco M, Feleppa M, Lipton RB, Rapoport AM, Bigal ME (2006) Mitochondrial dysfunction in migraine: evidence and hypotheses. Cephalalgia 26:361-372

15. Blake GJ, Ridker PM (2001) Novel clinical markers of vascular wall inflammation. Circ Res 89:763-771

16. Munno I, Centozone V, Marinaro M, Bassi A, Lacedra G, Causarano V, Nardelli P, Cassiano MA, Albano O (1998) Cytokines and migraine: increase of IK-5 an IL-4 plasma levels. Headache 38:465-467

17. Sarchielli P, Alberti A, Baldi A, Coppola F, Rossi C, Pierguidi L, Floridi A, Calabresi P (2006) Proinflammatory cytokines, adhesion molecules and lymphocyte integrin expression in the internal jugular blood of migraine patients without aura assessed ictally. Headache 46:200-207

18. Moskowitz MA, Macfarlane R (1993) Neurovascular and molecular mechanisms in migraine headaches. Cerebrovasc Brain Metab Rev 5:159-177

19. Welch KM (2003) Stroke and migraine-the spectrum of cause and effect. Funct Neurol 18:121-126

20. Gursoy-Ozdemir Y, Qiu J, Matsuoka N, Bolay H, Bermpohl D, Jin H, Wang X, Rosenberg GA, Lo EH, Moskowitz MA (2004) Cortical spreading depression activates and upregulates MMP-9. J Clin Invest 113:1447-1455

21. Lea RA, Ovcaric M, Sundholm J, MacMillan J, Griffiths LR (2004) The methylenetetrahydrofolate reductase gene variant C677T influences susceptibility to migraine with aura. BMC Med $2: 3$

22. Tietjen GE, Herial NA, Utley C, White L, Yerga-Woolwine S, Joe B (2009) Association of von Willebrand factor activity with
ACE I/D and MTHFR C677T polymorphisms in migraine. Cephalalgia 29:960-968

23. Scher AI, Terwindt GM, Picavet HS, Verschuren WM, Ferrari MD, Launer LJ (2005) Cardiovascular risk factors and migraine: the GEM population-based study. Neurology 64:614-620

24. Rao R, Rosati A, Liberini P, Gipponi S, Venturelli E, Sapia E, Ambrosi C, Padovani A (2008) Cerebrovascular risk factors and MRI abnormalities in migraine. Neurol Sci 29:S144-S145

25. Del Sette M, Dinia L, Bonzano L, Roccatagliata L, Finocchi C, Parodi RC, Sivori G, Gandolfo C (2008) White matter lesions in migraine and right-to-left shunt: a conventional and diffusion MRI study. Cephalalgia 28:376-382

26. Intiso D, Di Rienso F, Rinaldi G, Zarrelli MM, Giannatempo GM, Crociani P, Di Viesti P, Simone P (2006) Brain MRI white matter lesions in migraine patients: is there a relationship with antiphospholipid antibodies and coagulation parameters? Eur J Neurol 13:1364-1369

27. Headache Classificiation Subcommittee of the International Headache Society (2004) The international classification of headache disorders: 2nd edition. Cephalalgia 24(Suppl 1):9-160

28. Vermeer SE, Hollander M, van Dijk EJ, Hofman A, Koudstaal PJ, Breteler MMB (2003) Silent brain infarcts and white matter lesions increase stroke risk in general population. Stroke 34:1126-1129

29. Dalkara T, Nozari A, Moskowitz MA (2010) Migraine aura pathophysiology: the role of blood vessels and microembolisation. Lancet Neurol 9:309-317

30. Rocca MA, Colombo B, Inglese M, Codella M, Comi G, Filippi M (2003) A diffusion tensor magnetic resonance imaging study of brain tissue from patients with migraine. J Neurol Neurosurg Psychiatry 74:501-503

31. Valfrè W, Rainero I, Bergui M, Pinessi L (2008) Voxel-based morphometry reveals gray matter abnormalities in migraine. Headache 48:109-117

32. Aamodt AH, Stovner LJ, Hagen K, Bråthen G, Zwart J (2006) Headache prevalence related to smoking and alcohol use. The Head-HUNT Study. Eur J Neurol 13:1233-1238

33. López-Mesonero L, Márquez S, Parra P, Gámez-Leyva G, Muňoz P, Pascual J (2009) Smoking as a precipitating factor for migraine: a survey in medical students. J Headache Pain 10:101-103

34. Welch GN, Loscalzo J (1998) Mechanisms of disease: homocysteine and atherothrombosis. N Engl J Med 338:1042-1050

35. Stuhlinger MC, Stanger O (2005) Asymmetric dimethyl-L-arginine (ADMA): a possible link between homocyst(e)ine and endothelial dysfunction. Curr Drug Metab 6:3-14

36. Boger RH, Sydow K, Borlak J, Thum T, Lenzen H, Schubert B, Tsikas D, Bode-Boger SM (2000) LDL cholesterol upregulates synthesis of asymmetrical dimethylarginine in human endothelial cells: involvement of S-adenosylmethionine-dependent methyltransferases. Circ Res 87:99-105

37. Chrysohoou C, Pitsavos C, Skoumas J, Masoura C, Katinioti A, Panagiotakos D, Stefanadis C (2007) The emerging anti-inflammatory role of HDL-cholesterol, illustrated in cardiovascular disease free population; the ATTICA study. Int J Cardiol 122:29-33

38. Boysen G, Brander T, Christensen H, Gideon R, Truelsen T (2003) Homocysteine and risk of recurrent stroke. Stroke 34:1258-1261

39. Chambers JC, McGregor A, Jean-Marie J, Obeid OA, Kooner JS (1999) Demonstration of rapid onset vascular endothelial dysfunction after hyperhomocysteinemia: an effect reversible with vitamin C therapy. Circulation 99:1156-1160

40. Hering-Hanit R, Gadoth N, Yavetz A, Gavendo S, Sela B (2001) Is blood homocysteine elevated in migraine? Headache 41:779-781 
41. Hagen K, Bjøro T, Zwart JA, Vatten L, Stovner LJ, Bovim G (2001) Low headache prevalence amongst women with high TSH values. Eur J Neurol 8:693-699

42. Rainero I, Ferrero M, Rubino E, Valfrè W, Pellegrino M, Arvat E, Giordano R, Ghigo E, Limone P, Pinessi L (2006) Endocrine function is altered in chronic migraine patients with medicationoveruse. Headache 46:1309-1310

43. Squizzato A, Gerdes VEA, Brandjes DPM, Büller HR, Stam J (2005) Thyroid diseases and cerebrovascular disease. Stroke 36:2302-2310

44. Stone NJ (1994) Secondary causes of hyperlipidemia. Med Clin North Am 78:117-141

45. Bic Z, Blix GG, Hopp HP, Leslie FM, Schell MJ (1999) The influence of a low fat-diet on incidence and severity of migraine headaches. J Women's Health Gender Based Med 8:623-630
46. Heinecke JW, Kawamura M, Suzuki L, Chait A (1993) Oxidation of low-density lipoprotein by thiols: superoxide-dependent and independent mechanisms. J Lipid Res 34:2051-2061

47. Rapp JH, Pan XM, Neumann M, Hong M, Hollenbeck K, Liu J (2008) Microemboli composed of cholesterol crystals disrupt the blood-brain barrier and reduce cognition. Stroke 39:2354-2361

48. Weir CJ, Muir SW, Walters MR, Lees KR (2003) Serum urate as an independent predictor of poor outcome and vascular events after acute stroke. Stroke 34:1951-1957

49. Dawson J, Quinn TQ, Walter MR (2007) Xanthine oxidase inhibition-a new paradigm in management of cardiovascular risk. Curr Med Chem 14:1879-1886

50. Chamorro A, Obach V, Cervera A, Revilla M, Deulofeu R, Aponte JH (2002) Prognostic significance of uric acid serum concentration in patients with acute ischemic stroke. Stroke 33:1048-1052 